\title{
Multigap superconductivity in centrosymmetric and noncentrosymmetric rhenium-boron superconductors
}

\author{
T. Shang $\odot,{ }^{1, *}$ W. Xie,,${ }^{2, \dagger}$ J. Z. Zhao, ${ }^{3,4}$ Y. Chen, ${ }^{2}$ D. J. Gawryluk $\odot,{ }^{5}$ M. Medarde,${ }^{5}$ M. Shi, ${ }^{6}$ H. Q. Yuan, ${ }^{2}$ \\ E. Pomjakushina, ${ }^{5}$ and T. Shiroka ${ }^{7,8}$ \\ ${ }^{1}$ Key Laboratory of Polar Materials and Devices (MOE), School of Physics and Electronic Science, \\ East China Normal University, Shanghai 200241, China \\ ${ }^{2}$ Center for Correlated Matter and Department of Physics, Zhejiang University, Hangzhou 310058, China \\ ${ }^{3}$ Co-Innovation Center for New Energetic Materials, Southwest University of Science and Technology, Mianyang 621010, China \\ ${ }^{4}$ Research Laboratory for Quantum Materials, Singapore University of Technology and Design, Singapore 487372, Singapore \\ ${ }^{5}$ Laboratory for Multiscale Materials Experiments, Paul Scherrer Institut, CH-5232 Villigen, Switzerland \\ ${ }^{6}$ Swiss Light Source, Paul Scherrer Institut, CH-5232 Villigen, Switzerland \\ ${ }^{7}$ Laboratory for Muon-Spin Spectroscopy, Paul Scherrer Institut, CH-5232 Villigen, Switzerland \\ ${ }^{8}$ Laboratorium für Festkörperphysik, ETH Zürich, CH-8093 Zürich, Switzerland
}

(Received 22 February 2021; revised 8 April 2021; accepted 13 May 2021; published 28 May 2021)

\begin{abstract}
We report a comprehensive study of the centrosymmetric $\operatorname{Re}_{3} \mathrm{~B}$ and noncentrosymmetric $\operatorname{Re}_{7} \mathrm{~B}_{3}$ superconductors. At a macroscopic level, their bulk superconductivity $(\mathrm{SC})$, with $T_{c}=5.1 \mathrm{~K}\left(\operatorname{Re}_{3} \mathrm{~B}\right)$ and $3.3 \mathrm{~K}$ $\left(\operatorname{Re}_{7} \mathrm{~B}_{3}\right)$, was characterized via electrical-resistivity, magnetization, and heat-capacity measurements, while their microscopic superconducting properties were investigated by means of muon-spin rotation and relaxation $(\mu \mathrm{SR})$. In both $\operatorname{Re}_{3} \mathrm{~B}$ and $\mathrm{Re}_{7} \mathrm{~B}_{3}$ the low- $T$ zero-field electronic specific heat and the superfluid density (determined via transverse-field $\mu \mathrm{SR}$ ) suggest a nodeless SC. Both compounds exhibit some features of multigap SC, as evidenced by the temperature-dependent upper critical fields $H_{\mathrm{c} 2}(T)$, as well as by electronic band-structure calculations. The absence of spontaneous magnetic fields below the onset of SC, as determined from zero-field $\mu \mathrm{SR}$ measurements, indicates a preserved time-reversal symmetry in the superconducting state of both $\operatorname{Re}_{3} \mathrm{~B}$ and $\operatorname{Re}_{7} B_{3}$. Our results suggest that a lack of inversion symmetry and the accompanying antisymmetric spin-orbit coupling effects are not essential for the occurrence of multigap SC in these rhenium-boron compounds.
\end{abstract}

DOI: 10.1103/PhysRevB.103.184517

\section{INTRODUCTION}

The possibility to host unconventional and topological superconductivity (SC) or to act as systems to realize Majorana fermions [1-11] has made noncentrosymmetric superconductors (NCSCs) one of the most investigated materials in recent times. In NCSCs, a lack of inversion symmetry implies that admixtures of spin-singlet and spin-triplet superconducting pairings are allowed [1-3]. This sets the scene for a variety of exotic properties, such as upper critical fields beyond the Pauli limit $[12,13]$, nodes in the superconducting gap [14-17], and multigap SC [18]. More interestingly, by using the muon-spin relaxation ( $\mu \mathrm{SR})$ technique, time-reversal symmetry (TRS) breaking has been observed to occur in the superconducting state of selected weakly correlated NCSCs. These include CaPtAs [17], $\mathrm{LaNiC}_{2}$ [19], $\mathrm{La}_{7} T_{3}$ ( $T=$ transition metal) [20-22], $\mathrm{Zr}_{3} \operatorname{Ir}$ [23], and $\mathrm{Re} T$ [24-27]. Except for CaPtAs, where TRS breaking and superconducting gap nodes coexist below $T_{c}[17,28]$, in most other cases the superconducting properties resemble those of conventional superconductors, characterized by a fully opened energy gap. In general, the causes behind TRS breaking in these supercon-

\footnotetext{
*Corresponding author: tshang@phy.ecnu.edu.cn

${ }^{\dagger}$ Present address: DESY, Notkestraße 85, D-22607 Hamburg, Germany.
}

ductors are not yet fully understood and remain an intriguing open question.

To clarify the issue, $\alpha$-Mn-type $\operatorname{Re} T$ superconductors have been widely studied and demonstrated to show a superconducting state with broken TRS [24-27]. Our previous comparative $\mu$ SR studies on Re-Mo alloys, covering four different crystal structures (including the noncentrosymmetric $\alpha$-Mn type), revealed that the spontaneous magnetic fields occurring below $T_{c}$ were observed only in elementary rhenium and in $\operatorname{Re}_{0.88} \mathrm{Mo}_{0.12}[27,29,30]$. By contrast, TRS was preserved in the Re-Mo alloys with a lower Re content (below $\sim 88 \%$ ), independent of their centro- or noncentrosymmetric crystal structures [30]. Both elementary rhenium and $\mathrm{Re}_{0.88} \mathrm{Mo}_{0.12}$ adopting a simple centrosymmetric structure (hcp-Mg type) strongly suggests that a noncentrosymmetric structure is not essential in realizing TRS breaking in $\operatorname{Re} T$ superconductors. The $\mu$ SR results for the Re-Mo family, as well as other $\alpha$-Mn-type superconductors, e.g., $\mathrm{Mg}_{10} \mathrm{Ir}_{19} \mathrm{~B}_{16}$, $\mathrm{Nb}_{0.5} \mathrm{Os}_{0.5}, \mathrm{Re}_{3} \mathrm{~W}$, and $\mathrm{Re}_{3} \mathrm{Ta}$ [31-34], where TRS is preserved, clearly indicate that not only the Re presence but also its amount are crucial for the appearance and the extent of TRS breaking in $\operatorname{Re} T$ superconductors. How these results can be understood within a more general framework clearly requires further investigation.

Rhenium-boron compounds represent another suitable candidate system for studying the TRS breaking effects in the family of Re-based superconductors. Indeed, upon slight 
changes of the $\mathrm{Re} / \mathrm{B}$ ratio, both centrosymmetric $\mathrm{Re}_{3} \mathrm{~B}$ $\left(\mathrm{C}-\mathrm{Re}_{3} \mathrm{~B}\right)$ and noncentrosymmetric $\mathrm{Re}_{7} \mathrm{~B}_{3}\left(\mathrm{NC}-\mathrm{Re}_{7} \mathrm{~B}_{3}\right)$ compounds can be synthesized [35], the latter adopting the same $\mathrm{Th}_{7} \mathrm{Fe}_{3}$-type structure as $\mathrm{La}_{7} \mathrm{~T}_{3}$ superconductors [20-22], which frequently exhibit broken TRS in the superconducting state. Although selected properties of $\mathrm{Re}_{3} \mathrm{~B}$ and $\mathrm{Re}_{7} \mathrm{~B}_{3}$ have been investigated by different techniques [36-38], their superconducting properties at a microscopic level, in particular, the superconducting order parameter, require further investigation.

In this paper, we report on a comprehensive study of the superconducting properties of $\mathrm{Re}_{3} \mathrm{~B}$ and $\mathrm{Re}_{7} \mathrm{~B}_{3}$ carried out via electrical-resistivity, magnetization, heat-capacity, and muon-spin rotation and relaxation measurements, as well as by electronic band-structure calculations. Our endeavors serve a dual purpose. First, since $\mathrm{La}_{7} T_{3}$ shows evidence of TRS breaking below $T_{c}$, it is of interest to establish whether the isostructural $\operatorname{Re}_{7} \mathrm{~B}_{3}$ compound also displays similar features. Second, by systematically investigating the C- $\mathrm{Re}_{3} \mathrm{~B}$ and NC$\operatorname{Re}_{7} B_{3}$ superconductors, the previous findings regarding the $\operatorname{Re} T$ family can be extended also to other NCSC families, thus providing further insight into the open question of TRS breaking in NCSCs.

\section{EXPERIMENTAL AND NUMERICAL METHODS}

Polycrystalline rhenium-boron compounds were prepared by arc melting Re $(99.99 \%$, ChemPUR) and B (99.995\%, ChemPUR) powders with different stoichiometric ratios in a high-purity argon atmosphere. To improve the homogeneity, samples were flipped and remelted several times and, finally, annealed at $800^{\circ} \mathrm{C}$ for 2 weeks. The x-ray powder diffraction, measured using a Bruker D8 diffractometer with $\mathrm{Cu}$ $K \alpha$ radiation, confirmed the orthorhombic centrosymmetric structure of $\operatorname{Re}_{3} \mathrm{~B}(\mathrm{Cmcm}$, No. 63) and the hexagonal noncentrosymmetric structure of $\operatorname{Re}_{7} \mathrm{~B}_{3}\left(P 6_{3} m c\right.$, No. 186; see details in Fig. S1 in the Supplemental Material [39]). The magnetization, electrical-resistivity, and heat-capacity measurements were performed on Quantum Design magnetic property measurement system and physical property measurement system instruments, respectively. The bulk $\mu \mathrm{SR}$ measurements were carried out at the multipurpose surface-muon spectrometer (Dolly) of the Swiss muon source at Paul Scherrer Institut, Villigen, Switzerland. The $\mu$ SR data were analyzed by means of the MUSRFIT software package [40].

The electronic band structures of $\operatorname{Re}_{3} \mathrm{~B}$ and $\mathrm{Re}_{7} \mathrm{~B}_{3}$ were calculated via density functional theory (DFT), within the generalized gradient approximation (GGA) of the PerdewBurke-Ernzerhof realization [41], as implemented in QUANTUM ESPRESSO [42,43]. The projector augmented wave pseudopotentials were adopted for the calculation [44,45]. Electrons belonging to the outer atomic configuration were treated as valence electrons, here corresponding to 15 electrons in $\operatorname{Re}\left(5 s^{2} 5 p^{6} 5 d^{5} 6 s^{2}\right)$ and 3 electrons in $\mathrm{B}\left(2 s^{2} 2 p^{1}\right)$. The kinetic energy cutoff was fixed to 55 Ry. For the selfconsistent calculation, the Brillouin zone integration was performed on a $\Gamma$-centered mesh of $15 \times 15 \times 10 k$ points for $\mathrm{Re}_{3} \mathrm{~B}$ and $12 \times 12 \times 18 k$ points for $\mathrm{Re}_{7} \mathrm{~B}_{3}$. Experimentally determined lattice constants and atom positions were used in both calculations.

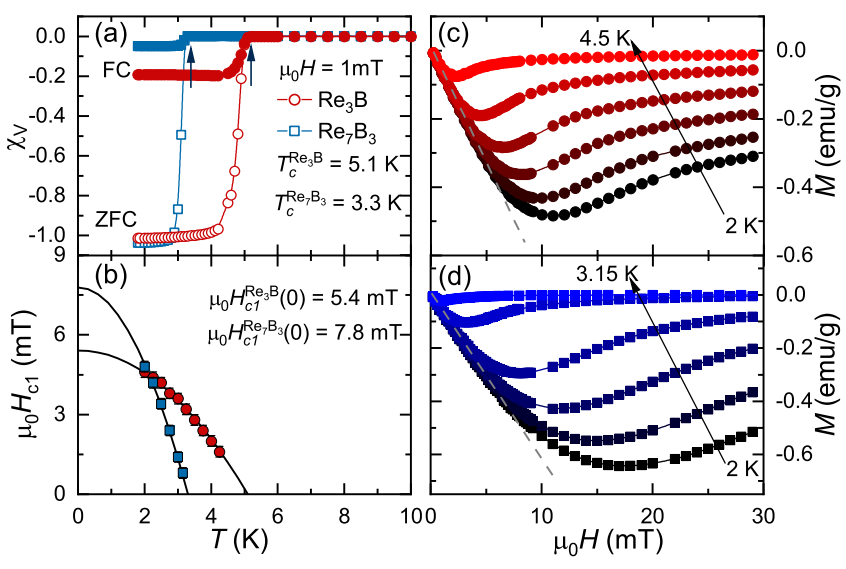

FIG. 1. (a) Temperature-dependent magnetic susceptibility of $\operatorname{Re}_{3} \mathrm{~B}$ and $\mathrm{Re}_{7} \mathrm{~B}_{3}$, measured in an applied field of $1 \mathrm{mT}$ using the ZFC and FC protocols. (b) Lower critical fields $H_{c 1}$ vs temperature. Solid lines are fits to $\mu_{0} H_{c 1}(T)=\mu_{0} H_{c 1}(0)\left[1-\left(T / T_{c}\right)^{2}\right]$. Field-dependent magnetization recorded at various temperatures for (c) $\operatorname{Re}_{3} \mathrm{~B}$ and (d) $\operatorname{Re}_{7} \mathrm{~B}_{3}$. For each temperature, $H_{c 1}$ was determined as the value where $M(H)$ starts deviating from linearity (see dashed lines).

\section{RESULTS AND DISCUSSION}

The bulk superconductivity of $C-\operatorname{Re}_{3} B$ and NC- $\operatorname{Re}_{7} B_{3}$ was first characterized by magnetic susceptibility measurements, using both field-cooled (FC) and zero-field-cooled (ZFC) protocols in an applied field of $1 \mathrm{mT}$. As indicated by the arrows in Fig. 1(a), a clear diamagnetic signal appears below the superconducting transition at $T_{c}=5.1$ and $3.3 \mathrm{~K}$ for $\operatorname{Re}_{3} \mathrm{~B}$ and $\operatorname{Re}_{7} B_{3}$, respectively. After accounting for the demagnetization factor, the superconducting shielding fraction of both samples is close to $100 \%$, indicative of bulk SC, which was further confirmed by heat-capacity measurements [39]. To determine the lower critical field $H_{c 1}$, essential for performing $\mu$ SR measurements on type-II superconductors, the field-dependent magnetization $M(H)$ was collected at various temperatures. Some representative $M(H)$ curves are shown in Figs. 1(c) and 1(d) for $\operatorname{Re}_{3} B$ and $\operatorname{Re}_{7} B_{3}$, respectively. The estimated $H_{c 1}$ values as a function of temperature are summarized in Fig. 1(b), resulting in $\mu_{0} H_{c 1}(0)=5.4(1) \mathrm{mT}$ and 7.8(1) $\mathrm{mT}$ for $\operatorname{Re}_{3} \mathrm{~B}$ and $\mathrm{Re}_{7} \mathrm{~B}_{3}$, respectively.

The upper critical field $H_{\mathrm{c} 2}$ of $\mathrm{Re}_{3} \mathrm{~B}$ and $\mathrm{Re}_{7} \mathrm{~B}_{3}$ was determined from measurements of the electrical resistivity, magnetization, and heat capacity under various magnetic fields up to $3 \mathrm{~T}$ (see Fig. S2 for details [39]). In zero magnetic field, the $T_{c}$ values determined using different methods are highly consistent. The upper critical fields are summarized in Figs. 2(a) and 2(b) versus the reduced superconducting transition temperature $T_{c} / T_{c}(0)$ for $\mathrm{Re}_{3} \mathrm{~B}$ and $\mathrm{Re}_{7} \mathrm{~B}_{3}$, respectively. $H_{\mathrm{c} 2}(T)$ was analyzed by means of Ginzburg-Landau (GL) [46], Werthamer-Helfand-Hohenberg (WHH) [47], and two-band (TB) models [48]. As shown in Fig. 2, both GL and WHH models can reasonably describe $H_{\mathrm{c} 2}(T)$ at low fields, i.e., $\mu_{0} H_{\mathrm{c} 2}<0.5 \mathrm{~T}(0.2 \mathrm{~T})$ for $\operatorname{Re}_{3} \mathrm{~B}\left(\mathrm{Re}_{7} \mathrm{~B}_{3}\right)$. However, at higher magnetic fields, both models deviate significantly from the experimental data and provide underestimated $H_{\mathrm{c} 2}$ values. Such a discrepancy most likely hints at multiple superconducting gaps in $\operatorname{Re}_{3} \mathrm{~B}$ and $\mathrm{Re}_{7} \mathrm{~B}_{3}$, as evidenced also by the 


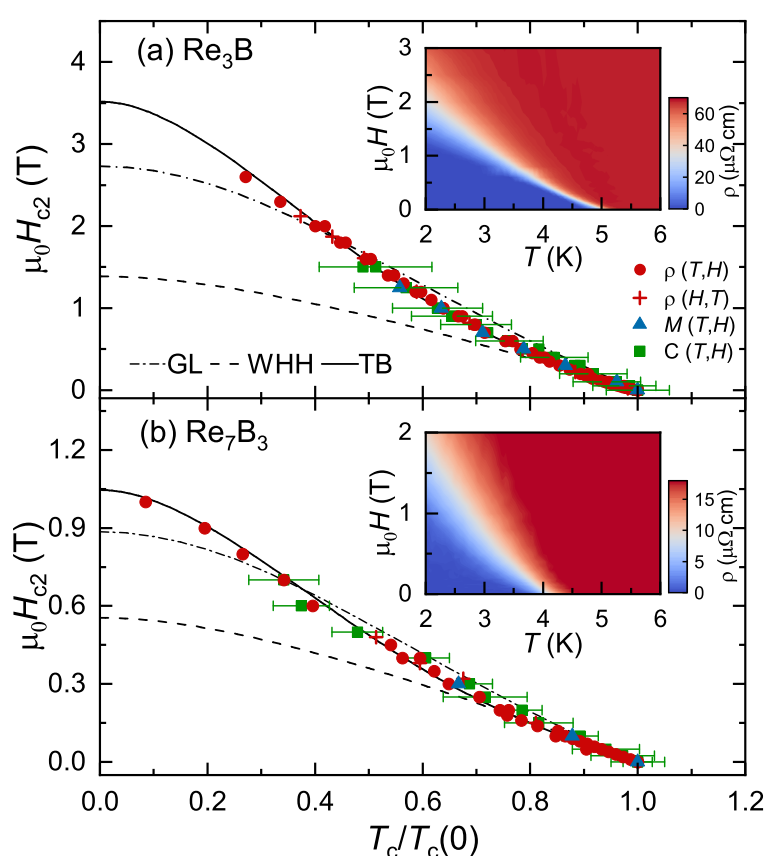

FIG. 2. Upper critical fields $H_{c 2}$ vs reduced temperature $T_{c} / T_{c}(0)$ for (a) $\operatorname{Re}_{3} \mathrm{~B}$ and (b) $\mathrm{Re}_{7} \mathrm{~B}_{3}$, as determined from temperaturedependent electrical resistivity $\rho(T, H)$, magnetization $M(T, H)$, and heat capacity $C(T, H)$ and from field-dependent electrical resistivity $\rho(H, T)$. The contour plots of $\rho(T, H)$ in the insets indicate a clear positive curvature close to $T_{c}$. Three different models, including the GL (dash-dotted line), WHH (dashed line), and TB models (solid line), were used to analyze the $H_{c 2}(T)$ data. Note the positive curvature visible near $\mu_{0} H \sim 0.5$ and $0.2 \mathrm{~T}$ for $\operatorname{Re}_{3} \mathrm{~B}$ and $\mathrm{Re}_{7} \mathrm{~B}_{3}$, respectively. The error bars refer to the superconducting transition widths $\Delta T_{c}$ in the specific-heat data.

positive curvature of $H_{\mathrm{c} 2}(T)$, a typical feature of multigap superconductors, as e.g., $\mathrm{Lu}_{2} \mathrm{Fe}_{3} \mathrm{Si}_{5}$ [49], $\mathrm{MgB}_{2}$ [50,51], and the recently reported $\mathrm{Mo}_{5} \mathrm{~PB}_{2}$ [52]. Physically, the positive curvature reflects the gradual suppression of the small superconducting gap upon increasing the magnetic field. As clearly demonstrated in the insets of Fig. 2, $H_{\mathrm{c} 2}(T)$ of $\mathrm{Re}_{3} \mathrm{~B}$ and $\mathrm{Re}_{7} \mathrm{~B}_{3}$ exhibit clear kinks close to 0.5 and $0.2 \mathrm{~T}$, respectively, most likely coinciding with the field values able to suppress the smaller gap. As shown by the solid lines in Fig. 2, the TB model shows remarkable agreement with the experimental data and provides $\mu_{0} H_{c 2}(0)=3.5(1)$ and $1.05(5) \mathrm{T}$ for $\operatorname{Re}_{3} \mathrm{~B}$ and $\operatorname{Re}_{7} B_{3}$, respectively.

To investigate at a microscopic level the $\mathrm{SC}$ of $\operatorname{Re}_{3} \mathrm{~B}$ and $\operatorname{Re}_{7} B_{3}$, we carried out systematic transverse-field (TF) $\mu$ SR measurements in an applied field of $20 \mathrm{mT}$, i.e., more than twice their $\mu_{0} H_{\mathrm{c} 1}(0)$ values [see Fig. 1(b)]. Representative $\mathrm{TF}-\mu \mathrm{SR}$ spectra collected in the superconducting and normal states of $\operatorname{Re}_{3} B$ and $\mathrm{Re}_{7} \mathrm{~B}_{3}$ are shown in Figs. 3(a) and 3(c), respectively. The additional field-distribution broadening due to the flux-line lattice (FLL) in the mixed state is clearly visible in Figs. 3(b) and 3(d), where the fast-Fourier transform (FFT) spectra of the corresponding TF- $\mu$ SR data are presented. To describe the asymmetric field distribution (e.g., see FFT at
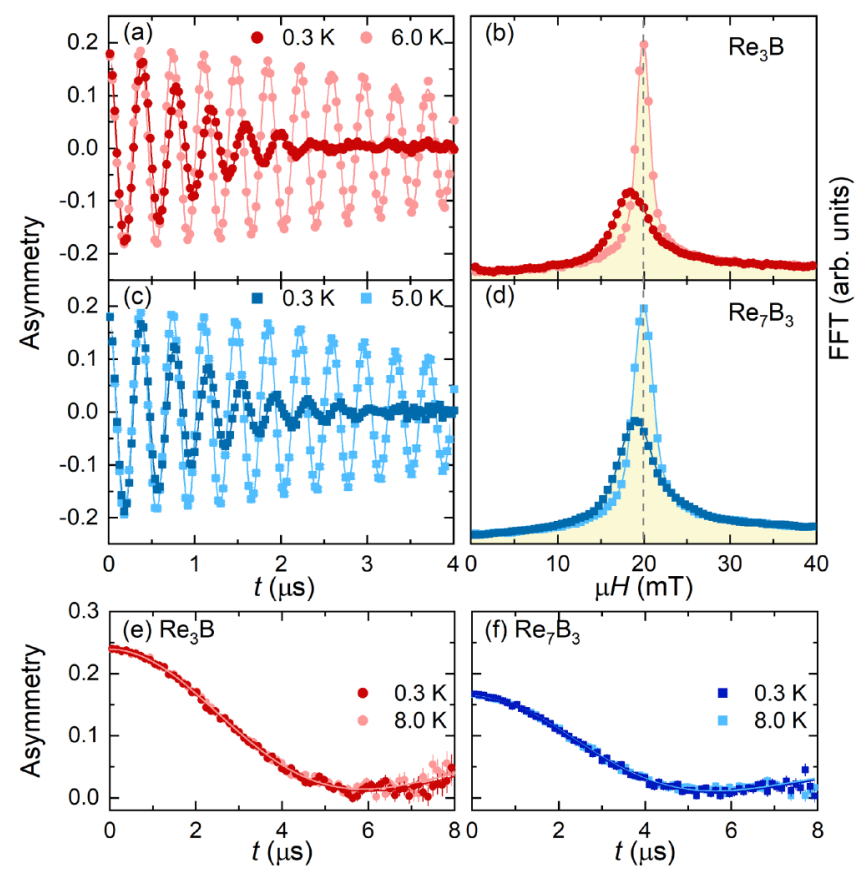

FIG. 3. (a) TF- $\mu$ SR spectra of $\mathrm{Re}_{3} \mathrm{~B}$ collected in the superconducting $(0.3 \mathrm{~K})$ and normal $(6 \mathrm{~K})$ states in an applied magnetic field of $20 \mathrm{mT}$. (b) Fast Fourier transforms of the TF- $\mu \mathrm{SR}$ data shown in (a). (c) and (d) The analogous results for the $\operatorname{Re}_{7} \mathrm{~B}_{3}$ case. The solid lines through the data are fits to Eq. (1), while the vertical dashed line marks the applied magnetic field. Note the clear diamagnetic shift and the field broadening at $0.3 \mathrm{~K}$, as shown in (b) and (d). ZF- $\mu \mathrm{SR}$ spectra of (e) $\operatorname{Re}_{3} \mathrm{~B}$ and (f) $\operatorname{Re}_{7} \mathrm{~B}_{3}$, collected in the superconducting and the normal states. Solid lines are fits using the equation described in the text. The overlapping data sets indicate no evident changes with temperature.

$0.3 \mathrm{~K})$, the TF- $\mu$ SR spectra were modeled using

$A(t)=\sum_{i=1}^{n} A_{i} \cos \left(\gamma_{\mu} B_{i} t+\phi\right) e^{-\sigma_{i}^{2} t^{2} / 2}+A_{\mathrm{bg}} \cos \left(\gamma_{\mu} B_{\mathrm{bg}} t+\phi\right)$.

Here $A_{i}, A_{\mathrm{bg}}$ and $B_{i}, B_{\mathrm{bg}}$ are the asymmetries and local fields sensed by implanted muons in the sample and sample holder (copper, which normally shows zero muon-spin depolarization), $\gamma_{\mu} / 2 \pi=135.53 \mathrm{MHz} / \mathrm{T}$ is the muon gyromagnetic ratio, $\phi$ is a shared initial phase, and $\sigma_{i}$ is the Gaussian relaxation rate of the $i$ th component. As shown by solid lines in Figs. 3(a) to 3(d), two oscillations (i.e., $n=2$ ) are required to properly describe the TF- $\mu \mathrm{SR}$ spectra for both $\operatorname{Re}_{3} \mathrm{~B}$ and $\operatorname{Re}_{7} \mathrm{~B}_{3}$. The derived $\sigma_{i}(T)$ as a function of temperature are summarized in the insets of Fig. 4. Above $T_{c}, \sigma_{i}(T)$ values are small and temperature independent, but below $T_{c}$ they start to increase due to the onset of FLL and the increased superfluid density. Simultaneously, a diamagnetic field shift appears below $T_{c}$, given by $\Delta B(T)=\langle B\rangle-B_{\text {appl. }}$, where $\langle B\rangle=\left(A_{1} B_{1}+A_{2} B_{2}\right) / A_{\text {tot }}, A_{\text {tot }}=A_{1}+A_{2}$, with $B_{\text {appl. }}$. being the applied field. The effective Gaussian relaxation rate can be estimated from $\sigma_{\text {eff }}^{2} / \gamma_{\mu}^{2}=\sum_{i=1}^{2} A_{i}\left[\sigma_{i}^{2} / \gamma_{\mu}^{2}+\left(B_{i}-\right.\right.$ $\left.\langle B\rangle)^{2}\right] / A_{\text {tot }}$ [53]. Considering the constant nuclear relaxation rate $\sigma_{\mathrm{n}}$ in the narrow temperature range investigated here, confirmed also by zero-field (ZF) $\mu$ SR measurements (see 


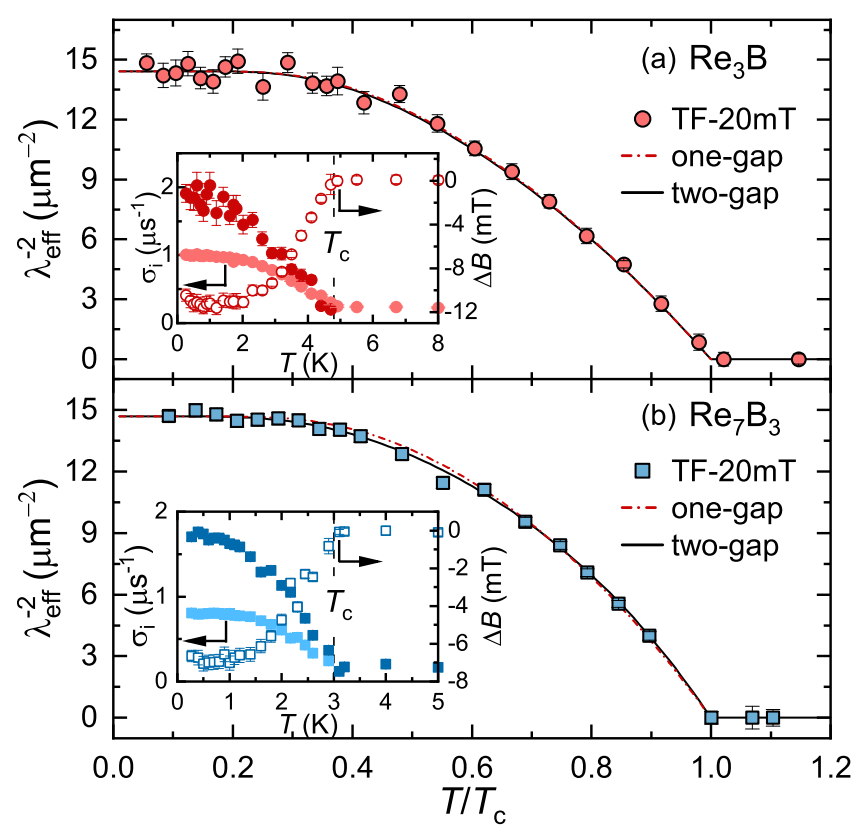

FIG. 4. Superfluid density vs reduced temperature $T / T_{c}$ for (a) $\operatorname{Re}_{3} \mathrm{~B}$ and (b) $\operatorname{Re}_{7} \mathrm{~B}_{3}$. The insets show the temperature-dependent muon-spin relaxation rate $\sigma_{\mathrm{i}}(T)$ (left axis) and the diamagnetic shift $\Delta B(T)$ (right axis). The dashed lines in the insets indicate the $T_{c}$ values, while the dash-dotted and solid lines in the main panels represent fits to a fully gapped $s$-wave model with one and two gaps, respectively.

details in Figs. 3(e) and 3(f) and Table S1 [39]), the superconducting contribution can be extracted using $\sigma_{\mathrm{sc}}=\sqrt{\sigma_{\mathrm{eff}}^{2}-\sigma_{\mathrm{n}}^{2}}$. Then, the effective magnetic penetration depth $\lambda_{\text {eff }}$ and, thus the superfluid density $\rho_{\mathrm{sc}}\left(\propto \lambda_{\text {eff }}^{-2}\right)$ can be calculated following $\sigma_{\mathrm{sc}}=0.172 \frac{\gamma_{\mu} \Phi_{0}}{2 \pi}(1-h)\left[1+1.21(1-\sqrt{h})^{3}\right] \lambda_{\text {eff }}^{-2}[54,55]$, where $h=H_{\text {appl }} / H_{\mathrm{c} 2}$, with $\mu_{0} H_{\text {appl }}=20 \mathrm{mT}$ being the applied magnetic field.

We also performed ZF- $\mu$ SR measurements in both the normal and the superconducting states of $\operatorname{Re}_{3} B$ and $\operatorname{Re}_{7} B_{3}$. As shown in Figs. 3(e) and 3(f), neither coherent oscillations nor fast decays could be identified in the spectra collected above $(8 \mathrm{~K})$ and below $T_{c}(0.3 \mathrm{~K})$, hence implying the lack of any magnetic order or fluctuations. The weak muonspin relaxation in the absence of an external magnetic field is mainly due to the randomly oriented nuclear moments, which can be modeled by means of a phenomenological relaxation function, consisting of a combination of Gaussian and Lorentzian Kubo-Toyabe relaxations [56,57], $A(t)=$ $A_{\mathrm{s}}\left[\frac{1}{3}+\frac{2}{3}\left(1-\sigma_{\mathrm{ZF}}^{2} t^{2}-\Lambda_{\mathrm{ZF}} t\right) e^{\left(-\frac{\sigma_{\mathrm{FF}}^{2} t^{2}}{2}-\Lambda_{\mathrm{ZF}} t\right)}\right]+A_{\mathrm{bg}}$. Here $A_{\mathrm{s}}(\equiv$ $\left.A_{\text {tot }}\right)$ and $A_{\text {bg }}$ are the same as in the TF- $\mu$ SR case [see Eq. (1)]. $\sigma_{\mathrm{ZF}}$ and $\Lambda_{\mathrm{ZF}}$ represent the zero-field Gaussian and Lorentzian relaxation rates, respectively. As shown by the solid lines in Figs. 3(e) and 3(f), the derived relaxations in the normal and the superconducting states are almost identical (see Table S1) [39], as confirmed also by the practically overlapping ZF- $\mu$ SR spectra above and below $T_{c}$. This lack of evidence for an additional $\mu \mathrm{SR}$ relaxation below $T_{c}$ excludes a possible TRS breaking in the superconducting state of both $\mathrm{C}-\mathrm{Re}_{3} \mathrm{~B}$ and $\mathrm{NC}-\mathrm{Re}_{7} \mathrm{~B}_{3}$.
The superfluid density $\rho_{\mathrm{sc}}$ vs the reduced $T / T_{c}$ is shown in Figs. 4(a) and 4(b) for $\operatorname{Re}_{3} B$ and $\operatorname{Re}_{7} B_{3}$, respectively. The temperature-independent superfluid density below $T_{c} / 3$ hints a fully gapped SC in both cases. Therefore, we analyzed $\rho_{\text {sc }}(T)$ by means of a fully gapped $s$-wave model:

$$
\rho_{\mathrm{sc}}(T)=\frac{\lambda_{\mathrm{eff}}^{-2}(T)}{\lambda_{0}^{-2}}=1+2 \int_{\Delta(T)}^{\infty} \frac{\partial f}{\partial E} \frac{E d E}{\sqrt{E^{2}-\Delta^{2}(T)}} .
$$

Here $f=\left(1+e^{E / k_{\mathrm{B}} T}\right)^{-1}$ and $\Delta(T)$ are the Fermi- and the superconducting-gap functions. $\Delta(T)$ is assumed to follow $\Delta(T)=\Delta_{0} \tanh \left\{1.82\left[1.018\left(T_{\mathrm{c}} / T-1\right)\right]^{0.51}\right\}[58]$, where $\Delta_{0}$ is the superconducting gap at $0 \mathrm{~K}$. Since the upper critical field $H_{\mathrm{c} 2}(T)$ exhibits typical features of multigap SC (see Fig. 2), the superfluid density was fitted using Eq. (2) with one and two gaps. In the two-gap case, $\rho_{\mathrm{sc}}(T)=w \rho_{\mathrm{sc}}^{\Delta^{\mathrm{f}}}(T)+$ $(1-w) \rho_{\mathrm{sc}}^{\Delta^{\mathrm{s}}}(T)$, with $\rho_{\mathrm{sc}}^{\Delta^{\mathrm{f}}}$ and $\rho_{\mathrm{sc}}^{\Delta^{\mathrm{s}}}$ being the superfluid densities related to the first $\left(\Delta^{\mathrm{f}}\right)$ and second $\left(\Delta^{\mathrm{s}}\right)$ gaps and $w$ being a relative weight. For $\operatorname{Re}_{3} B$, both the one- and two-gap models show an almost identical goodness-of-fit parameter $\left(\chi_{r}^{2} \sim 1.2\right)$, reflected in two practically overlapping fitting curves in Fig. 4(a). For $\mathrm{Re}_{7} \mathrm{~B}_{3}$, instead, the two-gap model $\left(\chi_{r}^{2} \sim 1.1\right)$ is slightly superior to the one-gap model $\left(\chi_{r}^{2} \sim\right.$ 2.2) [see Fig. 4(b)]. For the two-gap model, in the $\operatorname{Re}_{3} B$ case, the derived zero-temperature magnetic penetration depth is $\lambda_{0}=263(2) \mathrm{nm}$, and the gap values are $\Delta_{0}^{\mathrm{f}}=0.72(1) \mathrm{meV}$ and $\Delta_{0}^{\mathrm{s}}=0.87(2) \mathrm{meV}$, with a weight $w=0.7$. In the $\mathrm{Re}_{7} \mathrm{~B}_{3}$ case, the corresponding values are $\lambda_{0}=261(2) \mathrm{nm}, \Delta_{0}^{\mathrm{f}}=$ $0.35(1) \mathrm{meV}$, and $\Delta_{0}^{\mathrm{s}}=0.57(2) \mathrm{meV}$, with $w=0.27$. For the one-gap model, the gap values are $\Delta_{0}=0.77(2)$ and 0.50 (2) $\mathrm{meV}$ for $\mathrm{Re}_{3} \mathrm{~B}$ and $\mathrm{Re}_{7} \mathrm{~B}_{3}$, with the same $\lambda_{0}$ values as in the two-gap case.

Unlike in the clean-limit case [ $\xi_{0} \ll l_{\mathrm{e}}$; see Eq. (2)], in the dirty limit, the BCS coherence length $\xi_{0}$ is much larger than the electronic mean-free path $l_{\mathrm{e}}$. In the BCS approximation, the temperature-dependent superfluid density in the dirty limit is given by [59]

$$
\rho_{\mathrm{sc}}(T)=\frac{\Delta(T)}{\Delta_{0}} \tanh \left[\frac{\Delta(T)}{2 k_{\mathrm{B}} T}\right],
$$

where $\Delta(T)$ is the same as in Eq. (2). For $\operatorname{Re}_{3} \mathrm{~B}, \xi_{0}$ is larger than $l_{\mathrm{e}}\left(\xi_{0} / l_{\mathrm{e}} \sim 7.7\right)$; therefore, $\operatorname{Re}_{3} \mathrm{~B}$ belongs to the dirty limit. However, in $\operatorname{Re}_{7} \mathrm{~B}_{3}, \xi_{0}$ is smaller than $l_{\mathrm{e}}\left(\xi_{0} / l_{\mathrm{e}} \sim 0.3\right)$; therefore, it belongs to the clean limit. For both compounds, $\xi_{0}$ and $l_{\mathrm{e}}$ are not significantly different and exhibit similar magnitudes. Hence, both Eqs. (2) and (3) describe quite well the low- $T$ superfluid density and yield similar superconducting gaps (see Table I).

To further support the indications of a multigap SC obtained from $H_{c 2}$, we measured also the zero-field specific heat down to $T_{c} / 3$. After subtracting the phonon contribution $\left(\beta T^{2}+\delta T^{4}\right)$ from the measured data, the obtained electronic specific heat divided by $\gamma_{\mathrm{n}}$, i.e., $C_{\mathrm{e}} / \gamma_{\mathrm{n}} T$, is shown in Figs. 5(a) and 5(b) vs the reduced temperature $T / T_{c}$ for $\operatorname{Re}_{3} \mathrm{~B}$ and $\mathrm{Re}_{7} \mathrm{~B}_{3}$, respectively. The superconducting-phase contribution to the entropy can be calculated following the BCS expression [59]:

$$
S(T)=-\frac{6 \gamma_{\mathrm{n}}}{\pi^{2} k_{\mathrm{B}}} \int_{0}^{\infty}[f \ln f+(1-f) \ln (1-f)] d \epsilon,
$$


TABLE I. Normal- and superconducting-state properties of C$\mathrm{Re}_{3} \mathrm{~B}$ and $\mathrm{NC}-\mathrm{Re}_{7} \mathrm{~B}_{3}$, as determined from electrical-resistivity, magnetization, specific-heat, and $\mu \mathrm{SR}$ measurements, as well as from electronic band-structure calculations. The London penetration depth $\lambda_{\mathrm{L}}$, the effective mass $m^{\star}$, carrier density $n_{\mathrm{s}}$, BCS coherence length $\xi_{0}$, electronic mean free path $l_{\mathrm{e}}$, Fermi velocity $v_{\mathrm{F}}$, and effective Fermi temperature $T_{\mathrm{F}}$ were estimated following the methods in Ref. [30].

\begin{tabular}{|c|c|c|c|}
\hline Property & Units & $\operatorname{Re}_{3} \mathrm{~B}$ & $\operatorname{Re}_{7} B_{3}$ \\
\hline Space group & & $\mathrm{Cmcm}$ & $P 6_{3} m c$ \\
\hline Inversion center & & Yes & No \\
\hline$\rho_{0}$ & $\mu \Omega \mathrm{cm}$ & 68.0 & 18.5 \\
\hline Residual resistivity ratio & & 1.9 & 5.8 \\
\hline$T_{c}^{\rho}$ & $\mathrm{K}$ & 5.2 & 3.5 \\
\hline$T_{c}^{\chi}$ & $\mathrm{K}$ & 5.1 & 3.3 \\
\hline$T_{c}^{C}$ & $\mathrm{~K}$ & 4.7 & 3.1 \\
\hline$T_{c}^{\mu \mathrm{SR}}$ & $\mathrm{K}$ & 4.8 & 2.9 \\
\hline$\mu_{0} H_{c 1}$ & $\mathrm{mT}$ & $5.4(1)$ & $7.8(1)$ \\
\hline$\mu_{0} H_{c 2}$ & $\mathrm{~T}$ & $3.5(1)$ & $1.05(5)$ \\
\hline$\gamma_{n}$ & $\mathrm{~mJ} / \mathrm{mol} \mathrm{K}^{2}$ & $9.6(1)$ & $21.5(2)$ \\
\hline$\Theta_{\mathrm{D}}$ & $\mathrm{K}$ & $390(3)$ & $440(5)$ \\
\hline$N\left(\epsilon_{\mathrm{F}}\right)^{C}$ & states/eV f.u. & $4.1(1)$ & $9.1(1)$ \\
\hline$N\left(\epsilon_{\mathrm{F}}\right)^{\mathrm{DFT}}$ & states/eV f.u. & 2.35 & 5.7 \\
\hline$\Delta_{0}(C)$ & $\mathrm{meV}$ & $0.75(2)$ & $0.47(1)$ \\
\hline$\Delta_{0}(\mu \mathrm{SR})^{\text {clean }}$ & $\mathrm{meV}$ & $0.77(2)$ & $0.50(2)$ \\
\hline$\Delta_{0}(\mu \mathrm{SR})^{\text {dirty }}$ & $\mathrm{meV}$ & $0.66(2)$ & $0.44(1)$ \\
\hline$w$ & & 0.7 & 0.27 \\
\hline$\Delta_{0}^{f}(C)$ & $\mathrm{meV}$ & $0.69(2)$ & $0.32(1)$ \\
\hline$\Delta_{0}^{s}(C)$ & $\mathrm{meV}$ & $0.79(2)$ & $0.50(1)$ \\
\hline$\Delta_{0}^{f}(\mu \mathrm{SR})$ & $\mathrm{meV}$ & $0.72(1)$ & $0.35(1)$ \\
\hline$\Delta_{0}^{s}(\mu \mathrm{SR})$ & $\mathrm{meV}$ & $0.87(2)$ & $0.57(2)$ \\
\hline$\lambda_{0}$ & $\mathrm{~nm}$ & $263(2)$ & $261(2)$ \\
\hline$\lambda_{\mathrm{GL}}(0)$ & $\mathrm{nm}$ & $353(4)$ & $259(2)$ \\
\hline$\xi(0)$ & $\mathrm{nm}$ & $9.7(1)$ & $17.7(4)$ \\
\hline$\kappa$ & & $36(1)$ & $14.6(5)$ \\
\hline$\lambda_{\mathrm{L}}$ & $\mathrm{nm}$ & $90(5)$ & $229(2)$ \\
\hline$l_{\mathrm{e}}$ & $\mathrm{nm}$ & $2.2(1)$ & $22(1)$ \\
\hline$\xi_{0}$ & $\mathrm{~nm}$ & $17(1)$ & $6.4(1)$ \\
\hline$\xi_{0} / l_{\mathrm{e}}$ & & 7.7 & 0.3 \\
\hline$m^{\star}$ & $m_{e}$ & $7.0(2)$ & $10.4(1)$ \\
\hline$n_{\mathrm{s}}$ & $10^{28} \mathrm{~m}^{-3}$ & $2.4(3)$ & $0.56(1)$ \\
\hline$v_{\mathrm{F}}$ & $10^{5} \mathrm{~m} \mathrm{~s}^{-1}$ & $1.5(1)$ & $0.61(1)$ \\
\hline$T_{\mathrm{F}}$ & $10^{4} \mathrm{~K}$ & $1.0(1)$ & $0.25(1)$ \\
\hline
\end{tabular}

where $f$ is the same as in Eq. (2). Then, the temperaturedependent electronic specific heat below $T_{c}$ can be obtained from $C_{\mathrm{e}}(T)=T \frac{d S(T)}{d T}$. The dash-dotted lines in Fig. 5 represent fits of an $s$-wave model with $\gamma_{\mathrm{n}}=9.6(1)$ and $21.5(2) \mathrm{mJ} / \mathrm{mol} \mathrm{K}^{2}$ and a single gap $\Delta_{0}=0.75(2)$ and 0.47(1) meV for $\operatorname{Re}_{3} B$ and $\operatorname{Re}_{7} B_{3}$, respectively. For $\operatorname{Re}_{7} B_{3}$, while the one-gap model reproduces the data for $T / T_{c} \gtrsim 0.5$, it deviates from them at lower temperatures, hence yielding a slightly larger $\chi_{r}^{2} \sim 7.8$ than the two-gap model (see below). On the contrary, the two-gap model exhibits better agreement with the experimental data. The solid line in

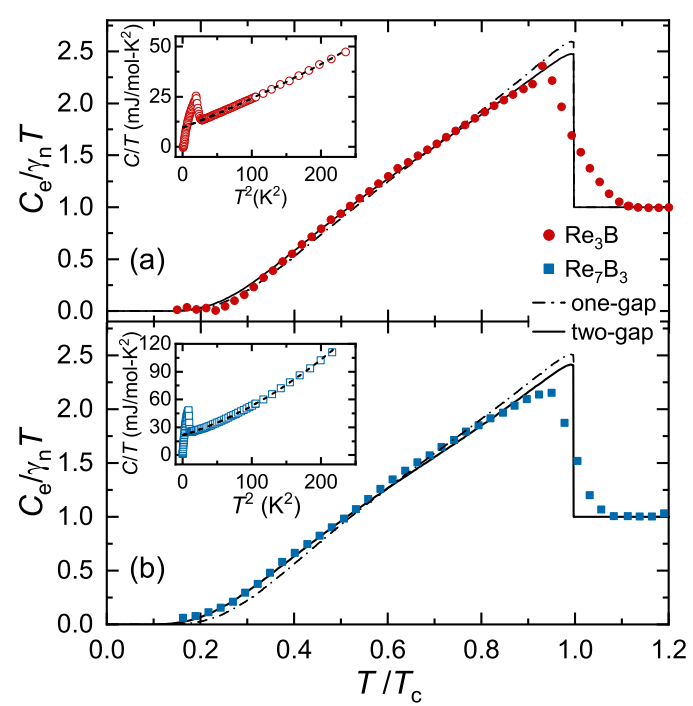

FIG. 5. Normalized electronic specific heat $C_{\mathrm{e}} / \gamma_{n} T$ versus reduced temperature $T / T_{c}$ for (a) $\operatorname{Re}_{3} \mathrm{~B}$ and (b) $\operatorname{Re}_{7} \mathrm{~B}_{3} \cdot \gamma_{n}$ is the normal-state electronic specific-heat coefficient. The insets show the measured specific heat $C / T$ versus $T^{2}$. The dashed lines in the insets are fits to $C / T=\gamma_{n}+\beta T^{2}+\delta T^{4}$ for $T>T_{c}$, while the dash-dotted and solid lines in the main panel represent the electronic specific heat calculated by considering a fully gapped $s$-wave model with one and two gaps, respectively.

Fig. 5(b) is a fit with two energy gaps, i.e., $C_{e}(T) / T=$ $w C_{e}^{\Delta^{\mathrm{f}}}(T) / T+(1-w) C_{e}^{\Delta^{\mathrm{s}}}(T) / T$. Here, each term represents a one-gap specific-heat contribution, with $w, \Delta^{\mathrm{f}}$, and $\Delta^{\mathrm{s}}$ being the same parameters as in the case of superfluid density fits. In $\operatorname{Re}_{7} \mathrm{~B}_{3}$, by sharing the $w$ values, the two-gap model gives $\Delta_{0}^{\mathrm{f}}=0.32(1) \mathrm{meV}$ and $\Delta_{0}^{\mathrm{s}}=0.50(1) \mathrm{meV}$, with $\chi_{r}^{2} \sim$ 1.7. Similarly, in $\operatorname{Re}_{3} B$, the solid line in Fig. $5(a)$ is a fit with $\Delta_{0}^{\mathrm{f}}=0.69(2) \mathrm{meV}$ and $\Delta_{0}^{\mathrm{s}}=0.79(2) \mathrm{meV}$. For $\mathrm{Re}_{3} \mathrm{~B}$, although the two-gap model agrees slightly better with the experimental data for $T / T_{c}>0.4$, below it, both one-gap and two-gap models deviate slightly from the measured data, probably reflecting an improper subtraction of the nuclear Schottky contribution (due to the limited lowest temperature that could be reached in this study; see details in Fig. S3 [39]). Measurements of zero-field specific heat down to the millikelvin range are clearly necessary to confirm the multigap nature of $\operatorname{Re}_{3} B$ and $\operatorname{Re}_{7} B_{3}$. Note that, for both compounds, the superconducting parameters determined from the specific heat and the TF- $\mu$ SR are remarkably consistent (see Table I).

Further evidence of the multigap SC and insight into the electronic properties of $\operatorname{Re}_{3} \mathrm{~B}$ and $\mathrm{Re}_{7} \mathrm{~B}_{3}$ comes from bandstructure calculations. The electronic band structures and the density of states (DOS) are shown in Fig. 6. As can be seen from Figs. 6(a) and 6(b), 4 and 12 different bands cross the Fermi level in $\operatorname{Re}_{3} \mathrm{~B}$ and $\mathrm{Re}_{7} \mathrm{~B}_{3}$, respectively. Close to $E_{\mathrm{F}}$, the DOS of both compounds is dominated by the Re $5 d$ orbitals, while the contribution from the B $2 p$ orbitals is negligible (see Figs. 6(a) and 6(b) and Fig. S4 [39]). Away from the Fermi level, the Re $5 d$ and B $2 p$ orbitals are highly hybridized. The estimated DOS at $E_{\mathrm{F}}$ are $\sim 4.7$ and $\sim 11.4$ states/(eV u.c.) for $\operatorname{Re}_{3} \mathrm{~B}$ and $\mathrm{Re}_{7} \mathrm{~B}_{3}$, both comparable to the experimental values determined from the electronic specific-heat coefficient (see 

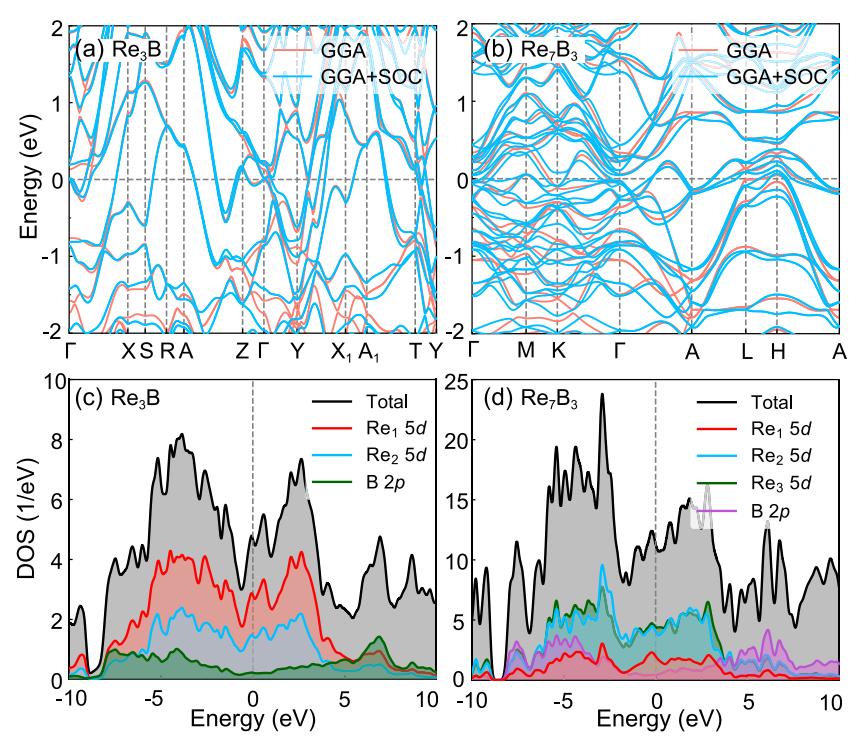

FIG. 6. Electronic band structure of $\mathrm{C}-\mathrm{Re}_{3} \mathrm{~B}$ (a) and $\mathrm{NC}-\mathrm{Re}_{7} \mathrm{~B}_{3}$ (b), calculated by ignoring (red) and by considering (blue) the spinorbit coupling. For both compounds, various bands which cross the Fermi level can be identified. The total and partial (Re $5 d$ and B $2 p$ orbitals) densities of states are shown in panels (c) and (d) for $\operatorname{Re}_{3} B$ and $\operatorname{Re}_{7} B_{3}$, respectively.

Table I). We expect the multigap features of $\operatorname{Re}_{3} B$ and $\operatorname{Re}_{7} B_{3}$ to be closely related to the different site symmetries of $\mathrm{Re}$ atoms in the unit cell. For $\operatorname{Re}_{3} \mathrm{~B}$, according to band-structure calculations, the contribution of $\operatorname{Re} 1(8 f)$ atoms to the DOS is comparable to that of $\operatorname{Re} 2(4 c)$ atoms [see Figs. 6(c)]. However, for $\operatorname{Re}_{7} \mathrm{~B}_{3}$, the contributions of $\operatorname{Re} 2(6 c)$ and $\operatorname{Re} 3$ (6c) atoms are preponderant compared to that of $\operatorname{Re} 1(2 b)$ atoms.

Now, let us compare the superconducting parameters of $\operatorname{Re}_{3} \mathrm{~B}$ and $\operatorname{Re}_{7} \mathrm{~B}_{3}$ with those of other superconductors. First, by using the $\mathrm{SC}$ parameters obtained from the measurements presented here, we calculated an effective Fermi temperature $T_{\mathrm{F}}=1.0(1) \times 10^{4}$ and $0.25(1) \times 10^{4} \mathrm{~K}$ for $\operatorname{Re}_{3} \mathrm{~B}$ and $\mathrm{Re}_{7} \mathrm{~B}_{3}$ (see other parameters in Table I). $T_{\mathrm{F}}$ is proportional to $n_{\mathrm{s}}^{2 / 3} / m^{\star}$, where $n_{\mathrm{s}}$ and $m^{\star}$ are the carrier density and the effective mass. Consequently, the different families of superconductors can be classified according to their $T_{c} / T_{\mathrm{F}}$ ratios into a so-called Uemura plot [60]. Several types of unconventional superconductors, including heavy fermions, organics, high- $T_{c}$ iron pnictides, and cuprates, all lie in a $10^{-2}<T_{c} / T_{\mathrm{F}}<10^{-1}$ band (gray region in Fig. S5 [39]). Conversely, many conventional superconductors, as e.g., $\mathrm{Al}, \mathrm{Sn}$, and $\mathrm{Zn}$, are located at $T_{c} / T_{\mathrm{F}} \lesssim 10^{-4}$. Between these two categories lie several multigap superconductors, e.g., $\mathrm{LaNiC}_{2}, \mathrm{Nb}_{5} \mathrm{Ir}_{3} \mathrm{O}, \mathrm{ReBe}_{22}$, $\mathrm{NbSe}_{2}$, and $\mathrm{MgB}_{2}$ [60-64]. Although there is no conclusive evidence for them to be classified as unconventional superconductors, the rhenium-boron superconductors lie clearly far off the conventional band. For $\operatorname{Re}_{3} \mathrm{~B}, T_{c} / T_{\mathrm{F}}=4.8 \times 10^{-4}$ is almost identical to the analogous value for multigap $\mathrm{ReBe}_{22}$ and $\mathrm{LaNiC}_{2}$, the latter representing a typical example of $\mathrm{NC}$ SCs. However, for $\operatorname{Re}_{7} \mathrm{~B}_{3}, T_{c} / T_{\mathrm{F}}=1.16 \times 10^{-3}$ is very close to the multigap $\mathrm{Nb}_{5} \mathrm{Ir}_{3} \mathrm{O}$ and elementary rhenium superconductors $[27,30,63]$, the latter showing a breaking of TRS in the superconducting state and exhibiting a centrosymmetric crystal structure. In general, most of the weakly correlated NCSCs, e.g., $\mathrm{ReT}, \mathrm{Mo}_{3} \mathrm{Al}_{2} \mathrm{C}, \mathrm{Li}_{2}(\mathrm{Pd}, \mathrm{Pt})_{3} \mathrm{~B}$, and $\mathrm{LaNiC}_{2}$, exhibit $T_{c} / T_{\mathrm{F}}$ values between the unconventional and conventional bands [64], and this is also the case for $\operatorname{Re}_{7} B_{3}$.

Second, we discuss why the multigap feature is more prominent in $\operatorname{Re}_{7} B_{3}$ than in $\operatorname{Re}_{3} B$, both in the temperaturedependent superfluid density and in the zero-field electronicspecific data. In general, if the weight of the second gap is relatively small and the gap sizes are not significantly different, it is difficult to discriminate between single- and two-gap superconductors based on temperature-dependent superconducting properties. For $\operatorname{Re}_{3} \mathrm{~B}$, the weight of the second gap $w=0.3$ is similar to that of $\operatorname{Re}_{7} \mathrm{~B}_{3}(w=0.27)$. However, the gap sizes are clearly distinct in $\operatorname{Re}_{7} \mathrm{~B}_{3}\left(\Delta^{\mathrm{f}} / \Delta^{\mathrm{s}} \sim 0.6\right)$ compared to $\operatorname{Re}_{3} \mathrm{~B}\left(\Delta^{\mathrm{f}} / \Delta^{\mathrm{s}} \sim 0.9\right)$. As a consequence, the multigap feature is more evident in $\operatorname{Re}_{7} \mathrm{~B}_{3}$. On the other hand, from the analysis of $H_{c 2}(T)$ using a two-band model, the derived interband and intraband couplings are $\lambda_{12}=0.08$ and $\lambda_{11} \sim \lambda_{22}=0.4$ and $\lambda_{12}=0.01$ and $\lambda_{11} \sim \lambda_{22}=0.15$ for $\operatorname{Re}_{3} \mathrm{~B}$ and $\operatorname{Re}_{7} \mathrm{~B}_{3}$, respectively. In both cases, the interband coupling is much smaller than the intraband coupling. In addition, the interband coupling of $\operatorname{Re}_{3} \mathrm{~B}$ (0.08) is larger than that of $\operatorname{Re}_{7} \mathrm{~B}_{3}$ (0.01). In such situation, the $\mathrm{SC}$ gaps open at different electronic bands, making the multigap features less distinguishable in the former case [65]. Despite these differences, the underlying multigap SC feature of both samples is reflected in their upper critical fields $H_{\mathrm{c} 2}(T)$ (see Fig. 2). To get further insight into the multigap $S C$ of $\operatorname{Re}_{3} B$ and $\operatorname{Re}_{7} B_{3}$, the measurements of the field-dependent superconducting Gaussian relaxation rate $\sigma_{\mathrm{sc}}(H)$ and of the electronic-specific heat coefficient $\gamma(H)$ provide a possible alternative, with both data sets being expected to show a distinct field response compared to a single-gap superconductor [52,61]. For example, $\gamma(H)$ exhibits a clear change in slope when the applied magnetic field suppresses the small gap, a feature recognized as the fingerprint of multigap superconductors. Conversely, $\gamma(H)$ is mostly linear in the single-gap case.

Finally, we discuss the effects of the lack of inversion symmetry in $\operatorname{Re}_{7} B_{3}$. In NCSCs, the occurrence of admixture of singlet and triplet pairings is allowed, whose mixing degree is generally believe to be related to the strength of the antisymmetric spin orbit coupling (ASOC) [1] and, thus, to unconventional SC. Here, by comparing $\mathrm{NC}-\mathrm{Re}_{7} \mathrm{~B}_{3}$ with $\mathrm{C}-\mathrm{Re}_{3} \mathrm{~B}$, we find that a noncentrosymmetric structure and its accompanying ASOC have little effect on the superconducting properties of $\operatorname{Re}_{7} \mathrm{~B}_{3}$. First, the upper critical field of $\mathrm{NC}$ $\operatorname{Re}_{7} B_{3}$ is three times smaller than that of $C-\operatorname{Re}_{3} B$; in both cases $H_{\mathrm{c} 2}$ is well below the Pauli limit. Second, according to the ZF- $\mu \mathrm{SR}$ data (Fig. 3), TRS is preserved in the superconducting states of both samples. The results presented here further support the idea that the presence of rhenium and its amount are the two key factors which determine the appearance of TRS breaking in Re-based superconductors, while the noncentrosymmetric structure plays only a marginal role. Obviously, the $\mathrm{Re}$ content in both $\mathrm{Re}_{3} \mathrm{~B}$ and $\mathrm{Re}_{7} \mathrm{~B}_{3}$ might be below a certain threshold value, e.g., $88 \%$ in Re-Mo alloys [30]. Therefore, it could be interesting to check, upon increasing the Re content, whether the TRS breaking effect will appear also in rhenium-boron superconductors and, if so, 
at which threshold value. Third, both $\operatorname{Re}_{7} \mathrm{~B}_{3}$ and $\mathrm{Re}_{3} \mathrm{~B}$ exhibit nodeless $\mathrm{SC}$ with multiple gaps. In the case of $\mathrm{Re}_{7} \mathrm{~B}_{3}$, whether the multigap feature is due to the band splitting caused by the ASOC or to the multiple bands crossing its Fermi level (the latter, in principle, accounting also for the $\mathrm{C}-\mathrm{Re}_{3} \mathrm{~B}$ case) requires further theoretical work. Overall, as can be seen from Fig. 6(b), the ASOC and the band splitting are relatively small in $\operatorname{Re}_{7} B_{3}$. Hence, we expect the spin-singlet pairing to be dominant in both the centrosymmetric and noncentrosymmetric rhenium-boron superconductors.

\section{CONCLUSION}

To summarize, we studied the superconducting properties of the centrosymmetric $\mathrm{Re}_{3} \mathrm{~B}$ and noncentrosymmetric $\operatorname{Re}_{7} \mathrm{~B}_{3}$ superconductors by means of electrical resistivity, magnetization, heat-capacity, and $\mu \mathrm{SR}$ techniques, as well as via numerical calculations. The superconducting states of $\operatorname{Re}_{3} \mathrm{~B}$ and $\mathrm{Re}_{7} \mathrm{~B}_{3}$ are characterized by $T_{c}=5.1$ and $3.3 \mathrm{~K}$, and upper critical fields $\mu_{0} H_{c 2}(0)=3.5$ and $1.05 \mathrm{~T}$, respectively. The temperature-dependent zero-field electronic specific heat and superfluid density reveal a nodeless superconductivity, well described by an isotropic s-wave model. Both $\operatorname{Re}_{3} \mathrm{~B}$ and $\operatorname{Re}_{7} \mathrm{~B}_{3}$ exhibit a positive curvature in their temperature-dependent upper critical field $H_{c 2}(T)$, an established fingerprint of multigap superconductors. By combining our extensive experimental results with numerical bandstructure calculations, we provided evidence of multigap superconductivity in both centro- and noncentrosymmetric rhenium-boron superconductors. Finally, the lack of spontaneous magnetic fields below $T_{c}$ indicates that, unlike in $\operatorname{Re} T$ or elementary rhenium, the time-reversal symmetry is preserved in the superconducting state of both $\operatorname{Re}_{3} \mathrm{~B}$ and $\operatorname{Re}_{7} \mathrm{~B}_{3}$. Our results suggest the spin-singlet paring channel to be dominant in rhenium-boron superconductors.

Note added. After the present manuscript was submitted, a related work by Sharma et al. [66] appeared, in which similar compounds were studied via the $\mu$ SR technique.

\section{ACKNOWLEDGMENTS}

This work was supported by the start funding from EastChina Normal University (ECNU), Swiss SNF Grants No. 200021-169455 and No. 206021-139082, and the Sino-Swiss Science and Technology Cooperation (Grant No. IZLCZ2170075). H.Q.Y. acknowledges support from the National Key R\&D Program of China (Grants No. 2017YFA0303100 and No. 2016YFA0300202), the Key R\&D Program of Zhejiang Province, China (Grant No. 2021C01002), and the National Natural Science Foundation of China (Grant No. 11974306). We acknowledge the allocation of beam time at the Swiss muon source.
[1] Non-centrosymmetric Superconductors, edited by E. Bauer and M. Sigrist, Lecture Notes in Physics, Vol. 847 (Springer, Berlin, 2012).

[2] M. Smidman, M. B. Salamon, H. Q. Yuan, and D. F. Agterberg, Superconductivity and spin-orbit coupling in noncentrosymmetric materials: A review, Rep. Prog. Phys. 80, 036501 (2017).

[3] S. K. Ghosh, M. Smidman, T. Shang, J. F. Annett, A. D. Hillier, J. Quintanilla, and H. Yuan, Recent progress on superconductors with time-reversal symmetry breaking, J. Phys.: Condens. Matter 33, 033001 (2021).

[4] H. Kim, K. Wang, Y. Nakajima, R. Hu, S. Ziemak, P. Syers, L. Wang, H. Hodovanets, J. D. Denlinger, P. M. R. Brydon, D. F. Agterberg, M. A. Tanatar, R. Prozorov, and J. Paglione, Beyond triplet: Unconventional superconductivity in a spin-3/2 topological semimetal, Sci. Adv. 4, eaao4513 (2018).

[5] Z. X. Sun, M. Enayat, A. Maldonado, C. Lithgow, E. Yelland, D. C. Peets, A. Yaresko, A. P. Schnyder, and P. Wahl, Dirac surface states and nature of superconductivity in noncentrosymmetric BiPd, Nat. Commun. 6, 6633 (2015).

[6] M. N. Ali, Q. D. Gibson, T. Klimczuk, and R. J. Cava, Noncentrosymmetric superconductor with a bulk three-dimensional Dirac cone gapped by strong spin-orbit coupling, Phys. Rev. B 89, 020505(R) (2014).

[7] M. Sato and S. Fujimoto, Topological phases of noncentrosymmetric superconductors: Edge states, Majorana fermions, and non-Abelian statistics, Phys. Rev. B 79, 094504 (2009).

[8] Y. Tanaka, Y. Mizuno, T. Yokoyama, K. Yada, and M. Sato, Anomalous Andreev Bound State in Noncentrosymmetric Superconductors, Phys. Rev. Lett. 105, 097002 (2010).
[9] M. Sato and Y. Ando, Topological superconductors: A review, Rep. Prog. Phys. 80, 076501 (2017).

[10] X.-L. Qi and S.-C. Zhang, Topological insulators and superconductors, Rev. Mod. Phys. 83, 1057 (2011).

[11] C. Kallin and J. Berlinsky, Chiral superconductors, Rep. Prog. Phys. 79, 054502 (2016).

[12] E. M. Carnicom, W. Xie, T. Klimczuk, J. J. Lin, K. Górnicka, Z. Sobczak, N. P. Ong, and R. J. Cava, $\mathrm{TaRh}_{2} \mathrm{~B}_{2}$ and $\mathrm{NbRh}_{2} \mathrm{~B}_{2}$ : Superconductors with a chiral noncentrosymmetric crystal structure, Sci. Adv. 4, eaar7969 (2018).

[13] E. Bauer, G. Hilscher, H. Michor, C. Paul, E. W. Scheidt, A. Gribanov, Y. Seropegin, H. Noël, M. Sigrist, and P. Rogl, Heavy Fermion Superconductivity and Magnetic Order in Noncentrosymmetric $\mathrm{CePt}_{3} \mathrm{Si}$, Phys. Rev. Lett. 92, 027003 (2004).

[14] H. Q. Yuan, D. F. Agterberg, N. Hayashi, P. Badica, D. Vandervelde, K. Togano, M. Sigrist, and M. B. Salamon, sWave Spin-Triplet Order in Superconductors without Inversion Symmetry: $\mathrm{Li}_{2} \mathrm{Pd}_{3} \mathrm{~B}$ and $\mathrm{Li}_{2} \mathrm{Pt}_{3} \mathrm{~B}$, Phys. Rev. Lett. 97, 017006 (2006).

[15] M. Nishiyama, Y. Inada, and G.-Q. Zheng, Spin Triplet Superconducting State Due to Broken Inversion Symmetry in $\mathrm{Li}_{2} \mathrm{Pt}_{3}$ B, Phys. Rev. Lett. 98, 047002 (2007).

[16] I. Bonalde, W. Brämer-Escamilla, and E. Bauer, Evidence for Line Nodes in the Superconducting Energy Gap of Noncentrosymmetric $\mathrm{CePt}_{3} \mathrm{Si}$ from Magnetic Penetration Depth Measurements, Phys. Rev. Lett. 94, 207002 (2005).

[17] T. Shang, M. Smidman, A. Wang, L.-J. Chang, C. Baines, M. K. Lee, Z. Y. Nie, G. M. Pang, W. Xie, W. B. Jiang, M. Shi, M. Medarde, T. Shiroka, and H. Q. Yuan, Simultaneous Nodal Superconductivity and Time-Reversal Symmetry Breaking in 
the Noncentrosymmetric Superconductor CaPtAs, Phys. Rev. Lett. 124, 207001 (2020).

[18] S. Kuroiwa, Y. Saura, J. Akimitsu, M. Hiraishi, M. Miyazaki, K. H. Satoh, S. Takeshita, and R. Kadono, Multigap Superconductivity in Sesquicarbides $\mathrm{La}_{2} \mathrm{C}_{3}$ and $\mathrm{Y}_{2} \mathrm{C}_{3}$, Phys. Rev. Lett. 100, 097002 (2008).

[19] A. D. Hillier, J. Quintanilla, and R. Cywinski, Evidence for Time-Reversal Symmetry Breaking in the Noncentrosymmetric Superconductor $\mathrm{LaNiC}_{2}$, Phys. Rev. Lett. 102, 117007 (2009).

[20] J. A. T. Barker, D. Singh, A. Thamizhavel, A. D. Hillier, M. R. Lees, G. Balakrishnan, D. M. Paul, and R. P. Singh, Unconventional Superconductivity in $\mathrm{La}_{7} \mathrm{Ir}_{3}$ Revealed by Muon Spin Relaxation: Introducing a New Family of Noncentrosymmetric Superconductor That Breaks Time-Reversal Symmetry, Phys. Rev. Lett. 115, 267001 (2015).

[21] D. Singh, M. S. Scheurer, A. D. Hillier, D. T. Adroja, and R. P. Singh, Time-reversal-symmetry breaking and unconventional pairing in the noncentrosymmetric superconductor $\mathrm{La}_{7} \mathrm{Rh}_{3}$, Phys. Rev. B 102, 134511 (2020).

[22] D. A. Mayoh, A. D. Hillier, G. Balakrishnan, and M. R. Lees, Evidence for the coexistence of time-reversal symmetry breaking and Bardeen-Cooper-Schrieffer-like superconductivity in $\mathrm{La}_{7} \mathrm{Pd}_{3}$, Phys. Rev. B 103, 024507 (2021).

[23] T. Shang, S. K. Ghosh, J. Z. Zhao, L.-J. Chang, C. Baines, M. K. Lee, D. J. Gawryluk, M. Shi, M. Medarde, J. Quintanilla, and T. Shiroka, Time-reversal symmetry breaking in the noncentrosymmetric $\mathrm{Zr}_{3} \mathrm{Ir}$ superconductor, Phys. Rev. B 102, 020503(R) (2020).

[24] R. P. Singh, A. D. Hillier, B. Mazidian, J. Quintanilla, J. F. Annett, D. M. Paul, G. Balakrishnan, and M. R. Lees, Detection of Time-Reversal Symmetry Breaking in the Noncentrosymmetric Superconductor $\mathrm{Re}_{6} \mathrm{Zr}$ using Muon-Spin Spectroscopy, Phys. Rev. Lett. 112, 107002 (2014).

[25] D. Singh, J. A. T. Barker, A. Thamizhavel, D. M. Paul, A. D. Hillier, and R. P. Singh, Time-reversal symmetry breaking in the noncentrosymmetric superconductor $\operatorname{Re}_{6} \mathrm{Hf}$ : Further evidence for unconventional behavior in the $\alpha$-Mn family of materials, Phys. Rev. B 96, 180501(R) (2017).

[26] T. Shang, G. M. Pang, C. Baines, W. B. Jiang, W. Xie, A. Wang, M. Medarde, E. Pomjakushina, M. Shi, J. Mesot, H. Q. Yuan, and T. Shiroka, Nodeless superconductivity and time-reversal symmetry breaking in the noncentrosymmetric superconductor $\mathrm{Re}_{24} \mathrm{Ti}_{5}$, Phys. Rev. B 97, 020502(R) (2018).

[27] T. Shang, M. Smidman, S. K. Ghosh, C. Baines, L. J. Chang, D. J. Gawryluk, J. A. T. Barker, R. P. Singh, D. M. Paul, G. Balakrishnan, E. Pomjakushina, M. Shi, M. Medarde, A. D. Hillier, H. Q. Yuan, J. Quintanilla, J. Mesot, and T. Shiroka, Time-Reversal Symmetry Breaking in Re-Based Superconductors, Phys. Rev. Lett. 121, 257002 (2018).

[28] W. Xie, P. R. Zhang, B. Shen, W. B. Jiang, G. M. Pang, T. Shang, C. Gao, M. Smidman, and H. Q. Yuan, CaPtAs: A new noncentrosymmetric superconductor, Sci. China: Phys., Mech. Astron. 63, 237412 (2020).

[29] T. Shang, D. J. Gawryluk, J. A. T. Verezhak, E. Pomjakushina, M. Shi, M. Medarde, J. Mesot, and T. Shiroka, Structure and superconductivity in the binary $\operatorname{Re}_{1-x} \mathrm{Mo}_{x}$ alloys, Phys. Rev. Mater. 3, 024801 (2019).

[30] T. Shang, C. Baines, L.-J. Chang, D. J. Gawryluk, E. Pomjakushina, M. Shi, M. Medarde, and T. Shiroka, $\operatorname{Re}_{1-x} \mathrm{Mo}_{x}$ as an ideal test case of time-reversal symmetry breaking in unconventional superconductors, npj Quantum Mater. 5, 76 (2020).

[31] A. A. Aczel, T. J. Williams, T. Goko, J. P. Carlo, W. Yu, Y. J. Uemura, T. Klimczuk, J. D. Thompson, R. J. Cava, and G. M. Luke, Muon spin rotation/relaxation measurements of the noncentrosymmetric superconductor $\mathrm{Mg}_{10} \mathrm{Ir}_{19} \mathrm{~B}_{16}$, Phys. Rev. B 82, 024520 (2010).

[32] D. Singh, J. A. T. Barker, A. Thamizhavel, A. D. Hillier, D. M. Paul, and R. P. Singh, Superconducting properties and $\mu \mathrm{SR}$ study of the noncentrosymmetric superconductor $\mathrm{Nb}_{0.5} \mathrm{Os}_{0.5}, \mathrm{~J}$. Phys.: Condens. Matter 30, 075601 (2018).

[33] P. K. Biswas, A. D. Hillier, M. R. Lees, and D. M. Paul, Comparative study of the centrosymmetric and noncentrosymmetric superconducting phases of $\mathrm{Re}_{3} \mathrm{~W}$ using muon spin spectroscopy and heat capacity measurements, Phys. Rev. B 85, 134505 (2012).

[34] J. A. T. Barker, B. D. Breen, R. Hanson, A. D. Hillier, M. R. Lees, G. Balakrishnan, D. M. Paul, and R. P. Singh, Superconducting and normal-state properties of the noncentrosymmetric superconductor $\operatorname{Re}_{3}$ Ta, Phys. Rev. B 98, 104506 (2018), and references therein .

[35] A. Kawano, Y. Mizuta, H. Takagiwa, T. Muranaka, and J. Akimitsu, The superconductivity in Re-B system, J. Phys. Soc. Jpn. 72, 1724 (2003).

[36] H. Takagiwa, A. Kawano, Y. Mizuta, T. Yamamoto, M. Yamada, K. Ohishi, T. Muranaka, J. Akimitsu, W. Higemoto, and R. Kadono, Magnetic penetration depth of a new boride superconductor $\mathrm{Re}_{3} \mathrm{~B}$, Phys. B (Amsterdam, Neth.) 326, 355 (2003).

[37] C. S. Lue, Y. F. Tao, and T. H. Su, Comparative NMR investigation of the Re-based borides, Phys. Rev. B 78, 033107 (2008).

[38] K. Matano, S. Maeda, H. Sawaoka, Y. Muro, T. Takabatake, B. Joshi, S. Ramakrishnan, K. Kawashima, J. Akimitsu, and G.-Q. Zheng, NMR and NQR studies on non-centrosymmetric superconductors $\mathrm{Re}_{7} \mathrm{~B}_{3}$, LaBiPt, and BiPd, J. Phys. Soc. Jpn. 82, 084711 (2013).

[39] See Supplemental Material at http://link.aps.org/supplemental/ 10.1103/PhysRevB.103.184517 for details on the measurements of crystal structure, electrical resistivity, magnetization, and heat capacity, as well as for the data analysis.

[40] A. Suter and B. M. Wojek, Musrfit: A free platformindependent framework for $\mu \mathrm{SR}$ data analysis, Phys. Procedia 30, 69 (2012).

[41] J. P. Perdew, K. Burke, and M. Ernzerhof, Generalized Gradient Approximation Made Simple, Phys. Rev. Lett. 77, 3865 (1996).

[42] P. Giannozzi et al., QUANTUM ESPRESSO: A modular and open-source software project for quantum simulations of materials, J. Phys.: Condens. Matter 21, 395502 (2009).

[43] P. Giannozzi et al., Advanced capabilities for materials modelling with Quantum ESPRESSO, J. Phys.: Condens. Matter 29, 465901 (2017).

[44] P. E. Blöchl, Projector augmented-wave method, Phys. Rev. B 50, 17953 (1994).

[45] A. D. Corso, Pseudopotentials periodic table: From $\mathrm{H}$ to $\mathrm{Pu}$, Comput. Mater. Sci. 95, 337 (2014).

[46] X. Zhu, H. Yang, L. Fang, G. Mu, and H.-H. Wen, Upper critical field, Hall effect and magnetoresistance in the ironbased layered superconductor $\mathrm{LaFeAsO}_{0.9} \mathrm{~F}_{0.1-\delta}$, Supercond. Sci. Technol. 21, 105001 (2008).

[47] N. R. Werthamer, E. Helfand, and P. C. Hohenberg, Temperature and purity dependence of the superconducting critical field, 
$H_{c 2}$. III. Electron spin and spin-orbit effects, Phys. Rev. 147, 295 (1966).

[48] A. Gurevich, Iron-based superconductors at high magnetic fields, Rep. Prog. Phys. 74, 124501 (2011), and references therein .

[49] Y. Nakajima, H. Hidaka, T. Nakagawa, T. Tamegai, T. Nishizaki, T. Sasaki, and N. Kobayashi, Two-band superconductivity featuring different anisotropies in the ternary iron silicide $\mathrm{Lu}_{2} \mathrm{Fe}_{3} \mathrm{Si}_{5}$, Phys. Rev. B 85, 174524 (2012).

[50] K.-H. Müller, G. Fuchs, A. Handstein, K. Nenkov, V. N. Narozhnyi, and D. Eckert, The upper critical field in superconducting $\mathrm{MgB}_{2}$, J. Alloys Compd. 322, L10 (2001).

[51] A. Gurevich, S. Patnaik, V. Braccini, K. H. Kim, C. Mielke, X. Song, L. D. Cooley, S. D. Bu, D. M. Kim, J. H. Choi, L. J. Belenky, J. Giencke, M. K. Lee, W. Tian, X. Q. Pan, A. Siri, E. E. Hellstrom, C. B. Eom, and D. C. Larbalestier, Very high upper critical fields in $\mathrm{MgB}_{2}$ produced by selective tuning of impurity scattering, Supercond. Sci. Technol. 17, 278 (2004).

[52] T. Shang, W. Xie, D. J. Gawryluk, R. Khasanov, J. Z. Zhao, M. Medarde, M. Shi, H. Q. Yuan, E. Pomjakushina, and T. Shiroka, Multigap superconductivity in the $\mathrm{Mo}_{5} \mathrm{~PB}_{2}$ boron-phosphorus compound, New J. Phys. 22, 093016 (2020).

[53] A. Maisuradze, R. Khasanov, A. Shengelaya, and H. Keller, Comparison of different methods for analyzing $\mu$ SR line shapes in the vortex state of type-II superconductors, J. Phys.: Condens. Matter 21, 075701 (2009), and references therein.

[54] E. H. Brandt, Properties of the ideal Ginzburg-Landau vortex lattice, Phys. Rev. B 68, 054506 (2003).

[55] W. Barford and J. M. F. Gunn, The theory of the measurement of the London penetration depth in uniaxial type-II superconductors by muon spin rotation, Phys. C (Amsterdam, Neth.) 156, 515 (1988).

[56] R. Kubo and T. Toyabe, A stochastic model for low-field resonance and relaxation, in Magnetic Resonance and Relaxation: Proceedings of the XIVth Colloque Ampère, edited by R. Blinc (North-Holland, Amsterdam, 1967), pp. 810-823.

[57] A. Yaouanc and P. D. de Réotier, Muon Spin Rotation, Relaxation, and Resonance: Applications to Condensed Matter (Oxford University Press, Oxford, 2011).
[58] A. Carrington and F. Manzano, Magnetic penetration depth of $\mathrm{MgB}_{2}$, Phys. C (Amsterdam, Neth.) 385, 205 (2003).

[59] M. Tinkham, Introduction to Superconductivity, 2nd ed. (Dover, Mineola, NY, 1996).

[60] Y. J. Uemura, L. P. Le, G. M. Luke, B. J. Sternlieb, W. D. Wu, J. H. Brewer, T. M. Riseman, C. L. Seaman, M. B. Maple, M. Ishikawa, D. G. Hinks, J. D. Jorgensen, G. Saito, and H. Yamochi, Basic Similarities among Cuprate, Bismuthate, Organic, Chevrel-Phase, and Heavy-Fermion Superconductors Shown by Penetration-Depth Measurements, Phys. Rev. Lett. 66, 2665 (1991).

[61] T. Shang, A. Amon, D. Kasinathan, W. Xie, M. Bobnar, Y. Chen, A. Wang, M. Shi, M. Medarde, H. Q. Yuan, and T. Shiroka, Enhanced $\mathrm{T}_{c}$ and multiband superconductivity in the fully-gapped $\mathrm{ReBe}_{22}$ superconductor, New J. Phys. 21, 073034 (2019).

[62] Y. J. Uemura, Twin spin/charge roton mode and superfluid density: Primary determining factors of $T_{c}$ in high- $T_{c}$ superconductors observed by neutron, ARPES, and $\mu$ SR, Phys. B (Amsterdam, Neth.) 374, 1 (2006).

[63] Y. Xu, S. Jöhr, L. Das, J. Kitagawa, M. Medarde, T. Shiroka, J. Chang, and T. Shang, Crossover from multiple- to single-gap superconductivity in $\mathrm{Nb}_{5} \mathrm{Ir}_{3-x} \mathrm{Pt}_{x} \mathrm{O}$ alloys, Phys. Rev. B 101, 134513 (2020).

[64] T. Shang, J. Philippe, J. A. T. Verezhak, Z. Guguchia, J. Z. Zhao, L.-J. Chang, M. K. Lee, D. J. Gawryluk, E. Pomjakushina, M. Shi, M. Medarde, H.-R. Ott, and T. Shiroka, Nodeless superconductivity and preserved time-reversal symmetry in the noncentrosymmetric $\mathrm{Mo}_{3} \mathrm{P}$ superconductor, Phys. Rev. B 99, 184513 (2019).

[65] V. G. Kogan, C. Martin, and R. Prozorov, Superfluid density and specific heat within a self-consistent scheme for a two-band superconductor, Phys. Rev. B 80, 014507 (2009).

[66] S. Sharma, Arushi, K. Motla, J. Beare, M. Nugent, M. Pula, T. J. Munsie, A. D. Hillier, R. P. Singh, and G. M. Luke, Fully gapped superconductivity in centrosymmetric and noncentrosymmetric Re-B compounds probed with $\mu \mathrm{SR}$, Phys. Rev. B 103, 104507 (2021). 